\title{
An Active and Structural Strain Model for Magnetostrictive Transducers
}

\author{
Marcelo J. Dapino ${ }^{1}$ Ralph C. Smith ${ }^{2}$ and Alison B. Flatau ${ }^{3}$ \\ ${ }^{1,3}$ AEEM Department, Iowa State University, Ames, IA 50011 \\ ${ }^{2}$ Center for Research in Scientific Computation, North Carolina State Univ., Raleigh, NC 27695
}

\begin{abstract}
We consider the modeling of strains generated by magnetostrictive materials in response to applied magnetic fields. The active or external component of the strain is due to the rotation of magnetic moments within the material to align with the applied field. This is characterized through consideration of the Jiles-Atherton mean field theory for ferromagnetic hysteresis in combination with a quadratic moment rotation model for magnetostriction. The second component of the strain reflects the passive or internal dynamics of the rod as it vibrates. This is modeled through force balancing which yields a wave equation with magnetostrictive inputs. The validity of a combined transducer model is illustrated through comparison with experimental data.
\end{abstract}

Keywords: Dynamic strain model, magnetostrictive materials, Terfenol-D

\section{Introduction}

This paper addresses the modeling of certain dynamics effects in magnetostrictive transducers. Such transducers utilize core materials, such as Terfenol-D, which have stoichiometries that promote both actuation and sensing. These capabilities are provided by the dual magnetostrictive effect; (i) stresses in the material produce measurable magnetic fields and (ii) the application of magnetic fields generates large strains in the material. By utilizing these properties, magnetostrictive transducers have been designed for applications ranging from ultrasonic transduction to vibration control in heavy structures.

At low to moderate drive levels, the magnetostrictive effects are relatively linear, and linear models and control laws have proven successful. At higher drive levels, however, the materials exhibit significant hysteresis and material nonlinearities which must be quantified before the materials can be employed to their full capacity. In dynamics applications, the materials also exhibit $\mathrm{AC}$ losses and resonance effects due to inherent magnetomechanical coupling within the material.

To illustrate issues which must be considered when modeling magnetostrictive transducers, we consider the prototypical transducer depicted in Figure 1. As detailed in [1], this design is typical for current control applications. The primary components of the actuator consist of a cylindrical magnetostrictive rod, a wound wire solenoid, an enclosing permanent magnet and a prestress mechanism. The rod is constructed so that magnetic moments are primarily oriented perpendicular to the longitudinal rod axis. The number of perpendicular moments and compression in the rod are further increased by the prestress mechanism. The application of current to the solenoid then produces a magnetic field which causes the moments to rotate so as to align with the field. The resulting strains and forces provide the actuator capabilities for the transducer. The capability for attaining bidirectional strains and forces is provided by a magnetic bias generated by either the surrounding permanent magnet or an applied DC current to the solenoid.

For control applications, it is necessary to accurately quantify the relationship between the current $I(t)$ applied to the solenoid and the strains $e(t)$ generated by the transducer. The strains contain two components which must

\footnotetext{
${ }^{1}$ Email: marcelod@iastate.edu; Telephone: 515-294-0088

${ }^{2}$ (Correspondence) - Email: rsmith@eos.ncsu.edu; Telephone: 919-515-7552

${ }^{3}$ Email: abf@iastate.edu; Telephone: 515-294-0094
} 
be modeled. Active or external strains, which are generated when moments in the material rotate to align with an applied field, provide the material with its actuator capabilities. We model this component in two steps. In the first, the Jiles-Atherton mean field theory for ferromagnetic hysteresis is modified to provide an energy-based relationship between the current $I(t)$ applied to the solenoid and the resulting magnetization $M$. A quadratic moment rotation model then yields the output magnetostriction $\lambda(t)$. As demonstrated in [2], the magnetostriction provides adequate fits to experimental strain data at low to moderate drive levels. At high input levels, however, it is inadequate since it incorporates only active strains and neglects material or passive strain effects. To model these effects, force balancing is used to derive a dynamic model quantifying the rod dynamics. This PDE model has the form of a wave equation with magnetostrictive inputs and boundary conditions which model the prestress mechanism. The solution to this system provides the rod displacements and corresponding total strains. As demonstrated through comparison with experimental data, this provides a model which characterizes the inherent magnetic hysteresis and accurately quantifies the strains and displacements output by the transducer.

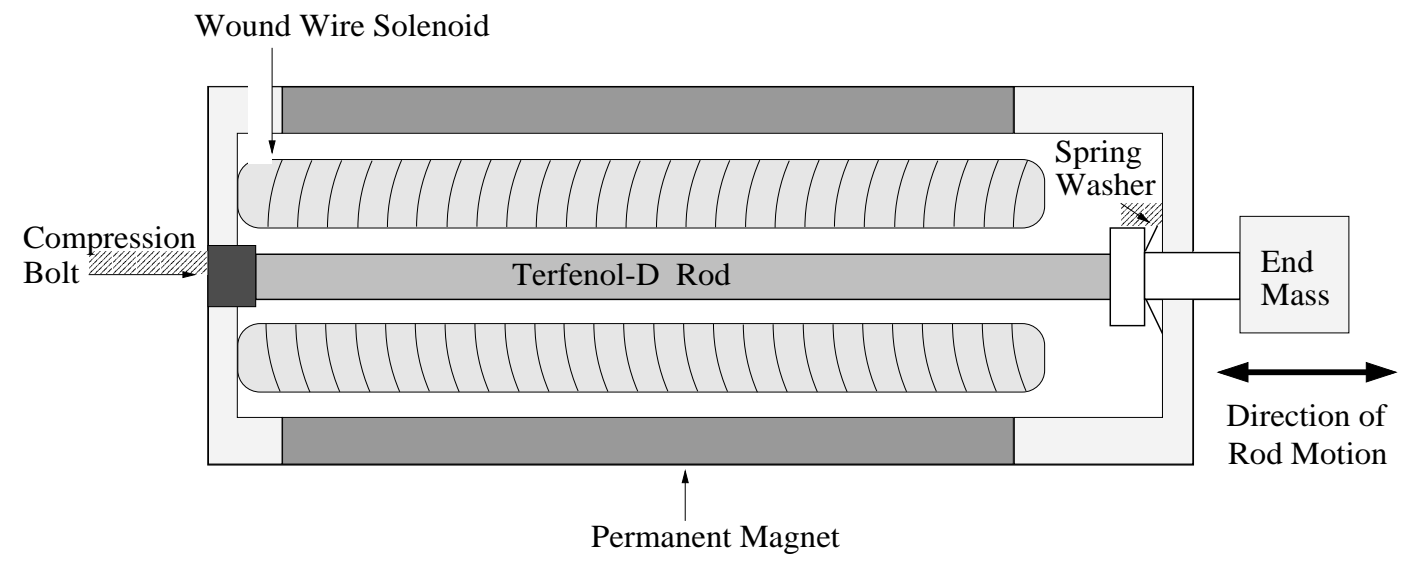

Figure 1. Cross section of a prototypical Terfenol-D magnetostrictive transducer.

\section{Magnetization and Magnetostriction Models}

The model which we employ for the magnetic component of the system is based upon the mean field theory of Jiles and Atherton [3, 4, 5, 6]. This theory is based upon the observation that moments in ferromagnetic materials, such as Terfenol-D, are highly aligned in regions termed domains at temperatures below the Curie point. Magnetization in such materials can then be described through the quantification of moment distributions within domains and in the transition regions between domains which are termed domain walls.

The reorientation of moments within domains produces a hysteresis-free (anhysteretic) magnetization $M_{a n}$ which is completely reversible. Such magnetization curves are rarely observed in laboratory materials, however, due to the presence of defects or second-phase materials (e.g., Dysprosium in Terfenol-D) which provide minimum energy states that impede domain wall movement and subsequent bulk moment reorientation. The theory of Jiles and Atherton quantifies the observed magnetization in terms of reversible $M_{r e v}$ and irreversible $M_{i r r}$ magnetization components defined by the domain wall energy. For low field variations about an equilibrium level, the magnetization is reversible since the domain walls remain pinned. At higher input levels, the walls attain sufficient energy to break the pinning sites (move out of the minimum energy state) and intersect remote pinning sites. This leads to an irreversible change in magnetization and provides a significant mechanism for hysteresis. The reader is referred to [3, 7] for additional details and discussion of other experimental phenomena, such as Barkhausen discontinuities, which are attributed to domain wall effects. This approach was initially employed in $[2,8,9]$ to model magnetostrictive transducers. We summarize here pertinent details and indicate extensions from the original model.

To quantify $M_{a n}, M_{r e v}$ and $M_{i r r}$, it is necessary to first determine the effective field $H_{\text {eff }}$ which acts upon magnetic moments in the Terfenol rod. As detailed in [3, 4], $H_{e f f}$ is dependent upon the magnetic field generated by the solenoid, magnetic moment interactions, crystal and stress anisotropies, temperature and the transducer 
architecture (e.g., end effects). In [2, 3], it is illustrated that for large prestresses, stress anisotropies dominate crystalline anisotropies; hence for this model, crystalline anisotropies are neglected. Under the assumption of fixed temperature and quasi-static operating conditions, the effective field is then modeled by

$$
H_{e f f}(t, x)=H(t, x)+\alpha M(t, x)+H_{\sigma}(t, x)
$$

where $x$ denotes the longitudinal coordinate. Here $H$ is the field generated by a solenoid with $n$ turns per unit length, $\alpha M$ quantifies the field due to magnetic interactions between moments, and $H_{\sigma}$ is the field due to magnetoelastic domain interactions. The parameter $\alpha$ quantifies the amount of domain interaction. For the prestress mechanism under consideration, it is demonstrated in [2] that the approximation $H_{\sigma}=\frac{9}{2} \frac{\lambda_{s} \sigma_{0}}{\mu_{0} M_{s}^{2}} M$ provides an adequate average of the stress contributions to the effective field. Here $\lambda_{s}$ and $M_{s}$ respectively denote the saturation magnetostriction and magnetization while $\mu_{0}$ is the free space permeability. The magnetic interactions and stress coefficient can then be combined into the single coefficient $\widetilde{\alpha}=\alpha+\frac{9}{2} \frac{\lambda_{s} \sigma_{0}}{\mu_{0} M_{s}^{2}}$ which must be estimated for a given system.

Empirical studies have indicated that under a variety of operating conditions, a reasonable approximation to the effective field is provided by

$$
H_{e f f}(t, x)=n I(t) \varphi(x)+\widetilde{\alpha} M(t)
$$

where $I(t)$ is the current to the solenoid and $\varphi(x)$ is an empirically-determined function which incorporates transducer anomalies, such as edge effects, that produce nonuniform field characteristics along the length of the rod. It should be noted that while the expression (1) is time-dependent, it must be restricted to low frequencies since the present model does not incorporate AC losses. The extension of this model to incorporate eddy current losses is under investigation.

For a computed effective field $H_{e f f}$, Boltzmann statistics are used to quantify the anhysteretic magnetization in terms of the Langevin function

$$
M_{a n}(t, x)=M_{s}\left[\operatorname{coth}\left(\frac{H_{e f f}(t, x)}{a}\right)-\frac{a}{H_{e f f}(t, x)}\right] .
$$

The constant $a=\frac{N k_{B} T}{\mu_{0} M_{s}}$, where $k_{B}$ is Boltzmann's constant, $N$ denotes the domain density and $k_{B} T$ represents the Boltzmann thermal energy, is treated as a parameter to be identified since $N$ is unknown.

As detailed in $[3,4]$, quantification of the energy required to break pinning sites then yields the expression

$$
\frac{\partial M_{i r r}}{\partial t}(t, x)=\frac{d I}{d t} \varphi(x) \cdot \frac{M_{a n}(t, x)-M_{i r r}(t, x)}{k \delta-\widetilde{\alpha}\left[M_{a n}(t, x)-M_{i r r}(t, x)\right] \frac{d M_{i r r}}{d M}}
$$

for the differential susceptibility of the irreversible magnetization curve. The constant $k$ has the form $k=\frac{\langle p\rangle\left\langle\varepsilon_{\pi}\right\rangle}{2 m \mu_{0}(1-c)}$, where $\langle p\rangle$ is the average density of pinning sites, $\left\langle\varepsilon_{\pi}\right\rangle$ is the average energy for $180^{\circ}$ walls, $c$ is a reversibility coefficient, and $m$ is the magnetic moment of a typical domain. This parameter provides a measure of the average energy required to break pinning sites and is also treated as a parameter to be estimated since $\langle p\rangle, c$ and $\left\langle\varepsilon_{\pi}\right\rangle$ are unknown. The parameter $\delta$ is defined to have the value +1 when $\frac{d H}{d t}>0$ and -1 when $\frac{d H}{d t}<0$ to guarantee that pinning always opposes changes in magnetization.

The reversible magnetization quantifies the degree to which domain walls bulge before attaining the energy necessary to break the pinning sites. To first approximation, the reversible magnetization is given by

$$
M_{r e v}(t, x)=c\left[M_{a n}(t, x)-M_{i r r}(t, x)\right]
$$

(see [4]). The reversibility coefficient $c$ can be estimated from the ratio of the initial and anhysteretic differential susceptibilities [5] or through a least squares fit to data.

The total magnetization is then given by

$$
M(t, x)=M_{r e v}(t, x)+M_{i r r}(t, x)
$$

where $M_{i r r}$ and $M_{r e v}$ are defined in (3) and (4) and the anhysteretic magnetization is given by (2). For implementation purposes, it is necessary to numerically integrate the expression (3) to obtain $M_{i r r}$. For the results in Section 4, this was accomplished via Euler's method. If higher accuracy is required, methods such as a trapezoid rule or Runge-Kutta method can be employed. 
The final aspect of the magnetization model concerns the characterization of the magnetostriction $\lambda \equiv \frac{d L}{L}$ in terms of the magnetization $M$. Because the magnetostriction indicates the relative change in length of the material from the ordered, but unaligned state, to the state in which domains are aligned, it provides a measure of the external strains generated by the material. As detailed in [3], consideration of the potential energy for the system yields

$$
\lambda(t, x)=\frac{3}{2} \frac{\lambda_{s}}{M_{s}^{2}} M^{2}(t, x)
$$

as a first approximation to the relationship between the magnetization and magnetostriction. Because $\lambda_{s}$ is dependent upon the operating conditions (e.g., the initial orientation of moments), it has sufficient variability to warrant estimation for individual transducers.

\section{Terfenol Rod Dynamics}

The expression (6) quantifies the external strains generated by the Terfenol rod in response to an applied current. In this section, we outline a model and approximation method for the rod dynamics with the strain $\lambda$ as an input. This provides a partially coupled model which incorporates both structural dynamics and magnetic hysteresis.

\subsection{Rod Model}

For modeling purposes, we consider a rod of length $L$ with cross section $A$ and longitudinal coordinate $x$. The density, Young's modulus and internal damping coefficient are denoted by $\rho, E$ and $c_{D}$, respectively, while $u(t, x)$ is used to denote inplane displacements in the longitudinal direction. The left end of the rod $(x=0)$ is assumed fixed while the prestress spring and attached mass at the opposite end are modeled by general elastic boundary conditions with an attached point mass.

Force balancing yields the modeling equation

$$
\rho A \frac{\partial^{2} u}{\partial t^{2}}=\frac{\partial N_{t o t}}{\partial x}
$$

where $N_{t o t}=N_{\text {int }}-N_{\text {ext }}$ denotes the general inplane force resultant. Under the assumption of small displacement, linear elasticity and Kelvin-Voigt damping, the passive (internal) and active (external) force resultants are given by

$$
\begin{aligned}
& N_{i n t}=E A \frac{\partial u}{\partial x}+c_{D} A \frac{\partial^{2} u}{\partial x \partial t} \\
& N_{e x t}=E A \lambda,
\end{aligned}
$$

respectively.

The fixed end condition is modeled by the boundary condition $u(t, 0)=0$. To obtain general elastic conditions at $x=L$, we consider an infinitesimal section from the rod end having the orientation depicted in Figure 2 . The attached mass is denoted by $M_{L}$ while the translation spring has stiffness $k_{L}$ and internal damping $c_{L}$. Force balancing for the infinitesimal edge element then yields the elastic boundary condition

$$
N_{\text {tot }}(t, L)+k_{L} u(t, L)+c_{L} \frac{\partial u}{\partial t}(t, L)=-M_{L} \frac{\partial^{2} u}{\partial t^{2}}(t, L)
$$

(a more general discussion regarding the derivation of general elastic boundary conditions can be found in [10]). When combined, the strong form of the model, with boundary conditions, is

$$
\begin{aligned}
& \rho A \frac{\partial^{2} u}{\partial t^{2}}=\frac{\partial N_{\text {tot }}}{\partial x} \\
& \left\{\begin{array}{l}
u(0, t)=0 \\
N_{\text {tot }}(L, t)=-k_{L} u(t, L)-c_{L} \frac{\partial u}{\partial t}(t, L)-M_{L} \frac{\partial^{2} u}{\partial t^{2}}(t, L) .
\end{array}\right.
\end{aligned}
$$

We point out that a similar modeling approach was employed by Hom and Shankar [11] for electrostrictive rods. They employ Fourier analysis to approximate the solution while we consider a corresponding weak formulation and finite element analysis to approximate the displacements. 
To define a weak or variational form of the model, the state $u$ is considered in the state space $X=L^{2}(0, L)$ and the space of test functions is taken to be $V=H_{L}^{1}(0, L) \equiv\left\{\phi \in H^{1}(0, L) \mid \phi(0)=0\right\}$. Multiplication by test functions followed by integration then yields the weak form

$$
\begin{aligned}
\int_{0}^{L} \rho A \frac{\partial^{2} u}{\partial t^{2}} \phi d x= & -\int_{0}^{L} E A \frac{\partial u}{\partial x} \frac{\partial \phi}{\partial x} d x-\int_{0}^{L} c_{D} A \frac{\partial^{2} u}{\partial x \partial t} \frac{\partial \phi}{\partial x} d x+\int_{0}^{L} E A \lambda \frac{\partial \phi}{\partial x} d x \\
& -\left[k_{L} u(t, L)+c_{L} \frac{\partial u}{\partial t}(t, L)+M_{L} \frac{\partial^{2} u}{\partial t^{2}}(t, L)\right] \phi(L)
\end{aligned}
$$

for all $\phi \in V$. It should be noted that in this form, displacements and test functions are differentiated only once compared with the second derivatives required in the strong form. This reduces the smoothness requirements on the finite element basis when constructing an approximation method. This structural model is summarized along with the previously-defined magnetization model in Algorithm 1.

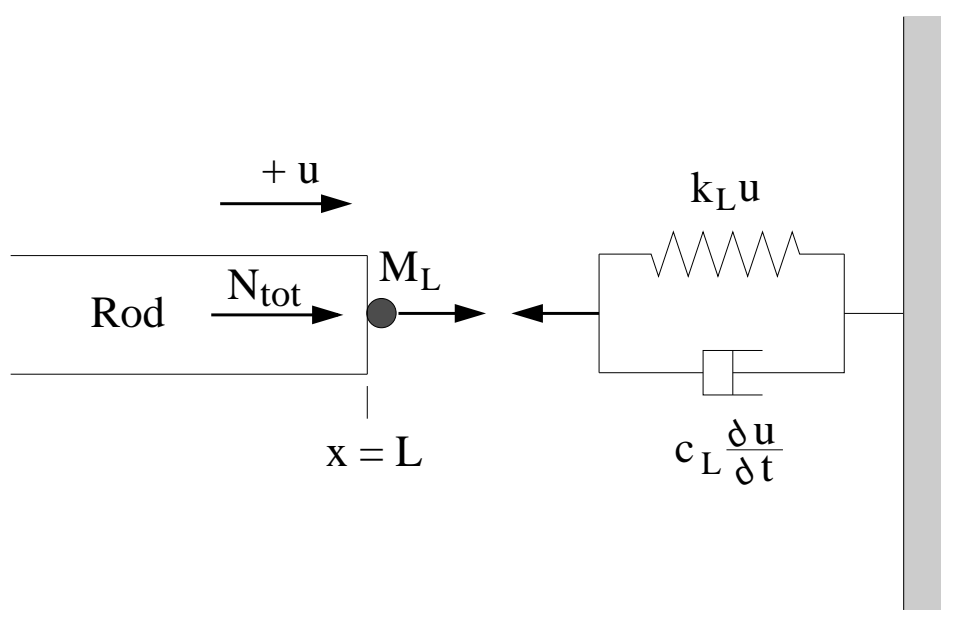

Figure 2. Orientation of spring forces, edge reactions and resultants for the Terfenol rod.

\subsection{Approximation Method}

To approximate the solution of (8), we consider a Galerkin discretization in space followed by a finite-difference approximation of the resulting temporal system. To this end, we consider a uniform partition of the interval $[0, L]$ with points $x_{i}=i h, i=0,1, \cdots, N$ and stepsize $h=L / N$ where $N$ denotes the number of subintervals. The spatial basis $\left\{\phi_{i}\right\}_{i=1}^{N}$ is comprised of linear splines, or 'hat functions,' of the form

$$
\begin{aligned}
& \phi_{i}(x)=\frac{1}{h}\left\{\begin{array}{l}
\left(x-x_{i-1}\right), x_{i-1} \leq x<x_{i} \\
\left(x_{i+1}-x\right), x_{i} \leq x \leq x_{i+1} \\
0, \text { otherwise }
\end{array} \quad, \quad i=1, \cdots, N-1\right. \\
& \phi_{N}(x)=\frac{1}{h}\left\{\begin{array}{l}
\left(x-x_{N-1}\right), x_{N-1} \leq x \leq x_{N} \\
0, \text { otherwise }
\end{array}\right.
\end{aligned}
$$

(see [12] for details). A general basis function $\phi_{i}$ and final basis function $\phi_{N}$ are plotted in Figure 3.

The solution $u(t, x)$ to $(8)$ is then approximated by the expansion

$$
u^{N}(t, x)=\sum_{j=1}^{N} u_{j}(t) \phi_{j}(x),
$$

in the subspace $H^{N}=\operatorname{span}\left\{\phi_{i}\right\}_{i=1}^{N}$. It should be noted that through the construction of the basis functions, the approximate solution satisfies $u^{N}(t, 0)=0$ and allows arbitrary displacements at $x=L$. 


\begin{tabular}{|c|c|}
\hline $\begin{array}{l}\text { External Mag- } \\
\text { netization and } \\
\text { Magnetostric- } \\
\text { tion }\end{array}$ & $\begin{array}{l}H(t, x)=n I(t) \varphi(x) \\
H_{e f f}(t, x)=H(t, x)+\widetilde{\alpha} M(t) \\
M_{a n}(t, x)=M_{s}\left[\operatorname{coth}\left(\frac{H_{e f f}(t, x)}{a}\right)-\left(\frac{a}{H_{e f f}(t, x)}\right)\right] \\
\frac{\partial M_{i r r}}{\partial t}(t, x)=n \frac{d I}{d t} \varphi(x) \cdot \frac{M_{a n}(t, x)-M_{i r r}(t, x)}{k \delta-\widetilde{\alpha}\left[M_{a n}(t, x)-M_{i r r}(t, x)\right] \frac{d M_{i r r}}{d M}} \\
M_{r e v}(t, x)=c\left[M_{a n}(t, x)-M_{i r r}(t, x)\right] \\
M(t, x)=M_{r e v}(t, x)+M_{i r r}(t, x) \\
\lambda(t, x)=\frac{3}{2} \frac{\lambda_{s}}{M_{s}^{2}} M^{2}(t, x)\end{array}$ \\
\hline $\begin{array}{l}\text { Dynamics of } \\
\text { Terfenol rod }\end{array}$ & $\begin{aligned} \int_{0}^{L} \rho A \frac{\partial^{2} u}{\partial t^{2}} \phi d x= & -\int_{0}^{L} E A \frac{\partial u}{\partial x} \frac{\partial \phi}{\partial x} d x-\int_{0}^{L} c_{D} A \frac{\partial^{2} u}{\partial x \partial t} \frac{\partial \phi}{\partial x} d x+\int_{0}^{L} E A \lambda \frac{\partial \phi}{\partial x} d x \\
& -\left[k_{L} u(t, L)+c_{L} \frac{\partial u}{\partial t}(t, L)+M_{L} \frac{\partial^{2} u}{\partial t^{2}}(t, L)\right] \phi(L)\end{aligned}$ \\
\hline
\end{tabular}

Algorithm 1. Time-dependent model quantifying the output magnetostriction $\lambda(t, x)$ and rod displacement $u(t, x)$.

A semi-discrete matrix system is obtained by considering the approximate solution $u^{N}(t, x)$ in (8) with the basis functions employed as test functions (this is equivalent to projecting the system (8) onto the finite dimensional subspace $H^{N}$ ). This yields the second-order time-dependent vector system

$$
M \ddot{\vec{u}}(t)+C \dot{\vec{u}}(t)+K \vec{u}(t)=\vec{f}(t)
$$

where $\vec{u}(t)=\left[u_{1}(t), \cdots, u_{N}(t)\right]$. The mass, stiffness and damping matrices have the components

$$
\begin{aligned}
& {[M]_{i j}=\left\{\begin{array}{l}
\int_{0}^{L} \rho A \phi_{i} \phi_{j} d x \quad, \quad i \neq n \text { and } j \neq n \\
\int_{0}^{L} \rho A \phi_{i} \phi_{j} d x+M_{L} \quad, \quad i=n \text { and } j=n
\end{array}\right.} \\
& {[K]_{i j}=\left\{\begin{array}{l}
\int_{0}^{L} E A \phi_{i}^{\prime} \phi_{j}^{\prime} d x \quad, \quad i \neq n \text { and } j \neq n \\
\int_{0}^{L} E A \phi_{i}^{\prime} \phi_{j}^{\prime} d x+k_{L} \quad, \quad i=n \text { and } j=n
\end{array}\right.} \\
& {[C]_{i j}=\left\{\begin{array}{l}
\int_{0}^{L} c_{D} A \phi_{i}^{\prime} \phi_{j}^{\prime} d x \quad, \quad i \neq n \text { and } j \neq n \\
\int_{0}^{L} c_{D} A \phi_{i}^{\prime} \phi_{j}^{\prime} d x+c_{L} \quad, \quad i=n \text { and } j=n
\end{array}\right.}
\end{aligned}
$$


while the force vector is defined by

$$
[\vec{f}(t)]_{i}=\int_{0}^{L} E A \lambda(t, x) \phi_{i}^{\prime}(x) d x
$$

(here' denotes a spatial derivative). With the definitions $\vec{y}(t)=[\dot{\vec{u}}(t), \vec{u}(t)]^{T}$,

$$
A=\left[\begin{array}{cc}
0 & I \\
-M^{-1} K & -M^{-1} C
\end{array}\right] \quad, \quad \vec{F}(t)=\left[\begin{array}{c}
0 \\
-M^{-1} \vec{f}(t)
\end{array}\right]
$$

the second-order system (9) can be posed as the first-order system

$$
\begin{aligned}
& \dot{\vec{y}}(t)=A \vec{y}(t)+\vec{F}(t) \\
& \vec{y}(0)=\vec{y}_{0}
\end{aligned}
$$

where the $2 N \times 1$ vector $\vec{y}_{0}$ denotes the projection of the initial conditions into the approximation space.

The system (10) must be discretized in time to permit numerical or experimental implementation. The choice of approximation method is dictated by accuracy and stability requirements, storage capabilities, sample rates, et cetera. A trapezoidal method was employed when experimentally implementing the method since it is moderately accurate, is A-stable, and requires minimal storage when implemented as a single step method. For temporal stepsizes $\Delta t$, a standard trapezoidal discretization yields the iteration

$$
\begin{aligned}
& \vec{y}_{j+1}=\mathcal{A} \vec{y}_{j}+\frac{1}{2} \mathcal{F}\left[\vec{F}\left(t_{j}\right)+\vec{F}\left(t_{j+1}\right)\right] \\
& \vec{y}_{0}=\vec{y}(0)
\end{aligned}
$$

where $t_{j}=j \Delta t$ and $\vec{y}_{j}$ appoximates $\vec{y}\left(t_{j}\right)$. The matrices

$$
\mathcal{A}=\left[I-\frac{\Delta t}{2} A\right]^{-1}\left[I+\frac{\Delta t}{2} A\right] \quad, \quad \mathcal{F}=\Delta t\left[I-\frac{\Delta t}{2} A\right]^{-1}
$$

need only be created once when numerically or experimentally implementing the method. This yields approximate solutions having $\mathcal{O}\left(h^{2},(\Delta t)^{2}\right)$ accuracy. For applications in which data at future times $t_{j+1}$ is unavailable, the algorithm (11) can be replaced by the modified trapezoidal iteration

$$
\begin{aligned}
& \vec{y}_{j+1}=\mathcal{A} \vec{y}_{j}+\mathcal{F} \vec{F}\left(t_{j}\right) \\
& \vec{y}_{0}=\vec{y}(0) .
\end{aligned}
$$

While this decreases slightly the temporal accuracy, for large sample rates with correspondingly small stepsizes $\Delta t$, the accuracy is still commensurate with that of the data.

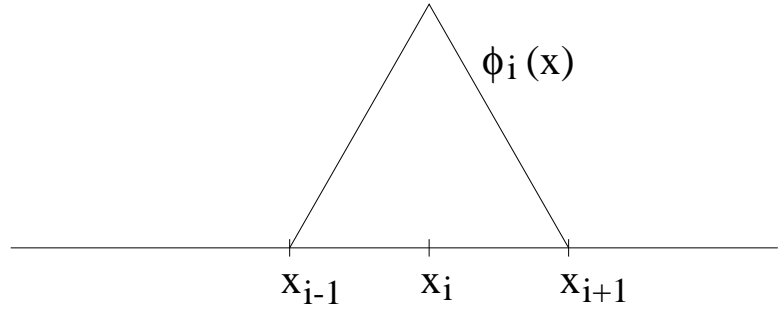

(a)

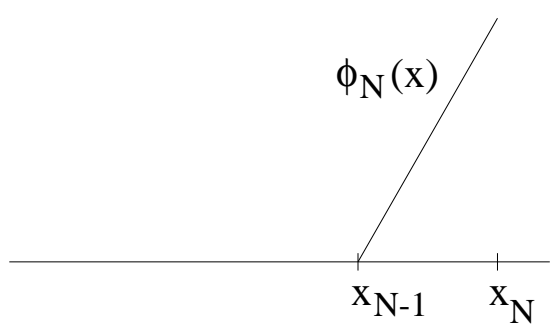

(b)

Figure 3. Linear basis functions (a) $\phi_{i}(x)$ and (b) $\phi_{N}(x)$. 


\section{Experimental Validation}

As summarized in Algorithm 1, the magnetization model (5), magnetostriction model (6) and PDE (8) can be combined to yield time-dependent displacement values of the Terfenol rod for all points along its length in response to an input current $I(t)$ to the solenoid. This model incorporates magnetic hysteresis, nonlinear strain properties and the coupling between the external strains generated by the material and the dynamics of the rod. In its present form, however, the model is not fully coupled since it does not yet incorporate the dynamic stress effects on the effective field and ensuing magnetization. This topic is under current investigation.

For the results which follow, experimental data was collected from a broadband transducer developed at Iowa State University (the general configuration of the transducer can be noted in Figure 1). A solid Terfenol-D ( $\left.\mathrm{Tb}_{0.3} \mathrm{Dy}_{0.7} \mathrm{Fe}_{1.9}\right)$ rod having a length of $115 \mathrm{~mm}(4.53 \mathrm{in})$ and diameter of $12.7 \mathrm{~mm}(0.5 \mathrm{in})$ was employed in the transducer. Mechanical prestresses to the rod were generated by a variable prestress bolt at one end of the transducer and Belleville washers fitted at the opposite end of the rod. Surrounding the rod were two coils consisting of an inner single layer 150-turn pickup coil and a multi-layer 900-turn drive coil. A current control amplifier (Techron 7780) provided the input to the drive coil to produce an applied AC magnetic field and DC bias as necessary. The reference signal to this amplifier was provided by a Tektronix spectrum analyzer and the applied magnetic field $H$ generated by the drive coil had a frequency of $0.7 \mathrm{~Hz}$ and magnitude up to $70 \mathrm{kA} / \mathrm{m}$.

A cylindrical permanent magnet surrounding the coils provided the capability for generating additional DC bias if necessary. This permanent magnet was constructed of Alnico V and was slit to reduce eddy current losses. Note that for the experiments reported here, biases generated in this manner were unnecessary and the permanent magnet was demagnetized to obtain unbiased data.

To determine a function $\varphi(x)$ (see (1)) which characterizes transducer anomalies, such as end effects, an axial Hall effect probe was used to map the flux density and corresponding field along the length of the rod. The resulting function $\varphi(x)$ is plotted in Figure 4 with the predominance of end effects readily noted.

The measured output from the transducer during operation included the current and voltage in the drive coil, the voltage induced in the pickup coil, and the mechanical output. The current $I(t)$ was used to compute the field $H(t, x)=n I(t) \varphi(x)$ applied to the rod. From the induced voltage in the pickup coil, the Faraday-Lenz law was used to compute the temporal derivative of the magnetic induction $B$. Integration then yielded the induction and magnetization $M=\frac{1}{\mu_{0}} B-H$. Both the field and magnetization were numerically filtered to remove the small biases due to instrumentation. A Lucas LVM-10 linear variable differential transformer, based upon changing reluctance, was used to measure the displacement of the rod tip. Corresponding strains at this point were then computed by dividing by the rod's length. The experimental data collected in this manner is plotted in Figures 5, 6 and 7 . Throughout the experiments, temperature was monitored using two thermocouples attached to the Terfenol-D sample and maintained within $5^{\circ} \mathrm{C}$ of the ambient temperature $\left(23^{\circ} \mathrm{C}\right)$.

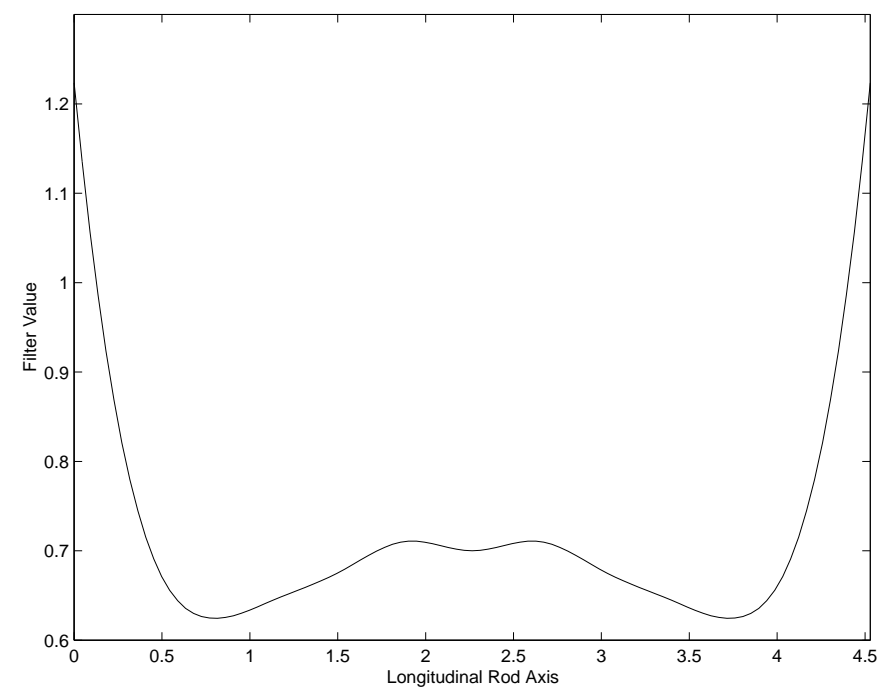

Figure 4. Empirical function $\varphi(x)$ used to qualify transducer effects. 
To employ the magnetization and structural model summarized in Algorithm 1, appropriate parameters must be ascertained. These include the magnetization parameters $\widetilde{\alpha}, c, k, a, \lambda_{s}, M_{s}$, the structural parameters $\rho, E, c_{D}$, the spring constants $k_{L}, c_{L}$ and the end mass $M_{L}$. The values used here are summarized in Table 1 . The magnetization parameters were estimated through a least squares fit to data as detailed in [2]. The values of $\rho$ and $E$ are published specifications for Terfenol-D while the damping parameters $c_{D}$ and $c_{L}$ were chosen within a range typical for the material. The spring stiffness coefficient $k_{L}$ was measured through a compression test while the end mass $M_{L}$ was measured directly. We note that while the specification of parameters in this manner provided adequate model fits, they are not optimal. To obtain optimal parameters and corresponding model fits, it is necessary to estimate all parameters through a least squares fit to data.

The model summarized in Algorithm 1 characterizes two aspects of the strain for the Terfenol rod. The magnetostriction $\lambda$ quantifies the external or active component of the strain while $e=\frac{\partial u}{\partial x}$ denotes the total strain for the rod (the combination of active and passive strains). It is necessary to consider the displacements $u$ and total strain $e$ when modeling the transducer core since these are the quantities measured in experiments. To illustrate, the magnetostriction $\lambda$ and strain $e$ given by the finite element approximation (11) to the model in Algorithm 1 are compared with experimental data in Figure 5. In both cases, the field $H$ measured at the drive coil was filtered with the function $\varphi$ depicted in Figure 4 and then used to compute the magnetization.

It is observed that some discrepancy occurs in both the magnetostriction and strain due to limitations in the quadratic model (6). The total strain provided by the dynamic model does, however, include the hysteresis observed in the experimental data. This is a significant advantage over the modeled magnetostriction which is single-valued. This leads to the highly accurate model fit observed in Figure 6 where the relation between the input field $H$ and output strain is considered.

For comparison purposes, we include in Figure 7 the corresponding model fits obtained when the filtering function $\varphi(x)$ was omitted and direct field measurements were used to compute $M$ and $\lambda$. This was the regime considered in [2] where it was demonstrated that use of magnetostriction rather than total strain led to an adequate model at low to moderate drive levels but provided inadequate fits at high input levels. These conclusions are reiterated by the model fits observed in Figure 7. It is noted that even when the saturation magnetostriction was scaled to the maximum experimentally observed value, the lack of hysteresis in the relation between $M$ and $\lambda$ led to an inadequate characterization of $\lambda$ in terms of $H$. A comparison with Figures $5 \mathrm{~b}$ and $6 \mathrm{~b}$ again indicates the necessity of considering the total strains.

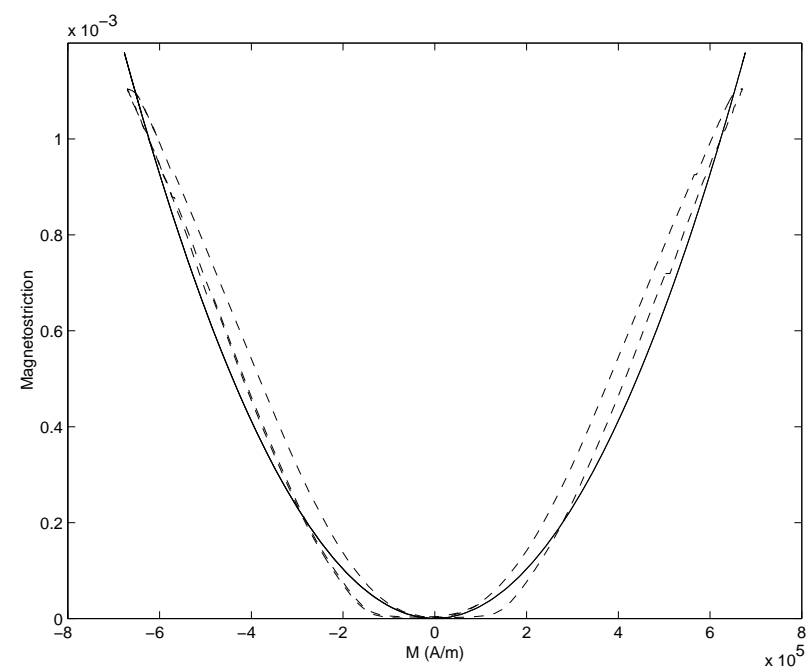

(a)

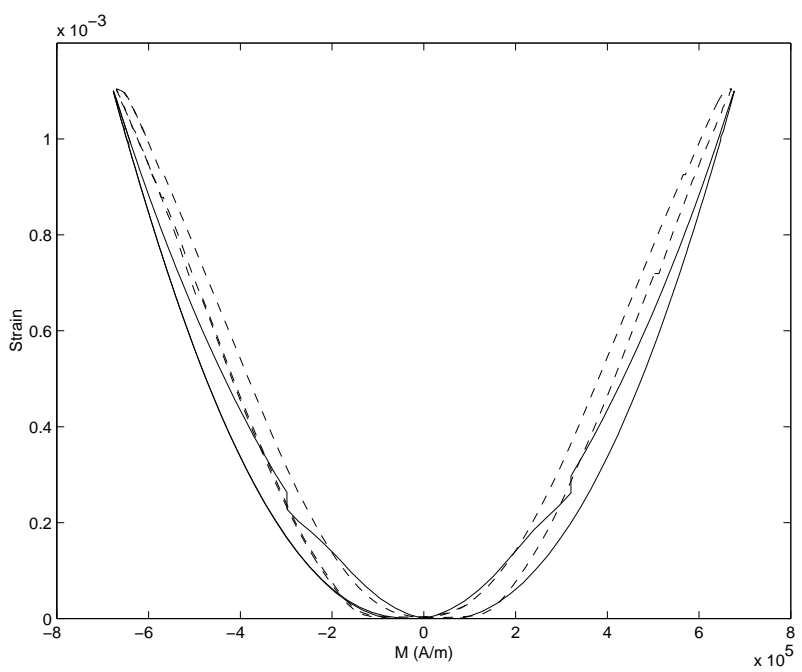

(b)

Figure 5. Experimental data (-- ) and model dynamics (- ) with the magnetization $M$ computed using filtered magnetic field data $H(t, x)=n I(t) \varphi(x)$; (a) Magnetostriction $\lambda$, (b) Total strain $e$. 


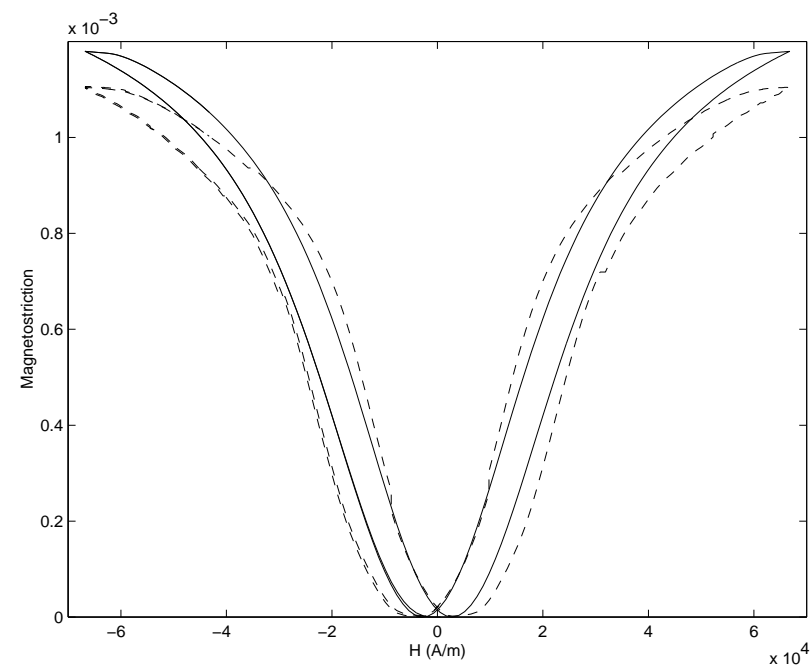

(a)

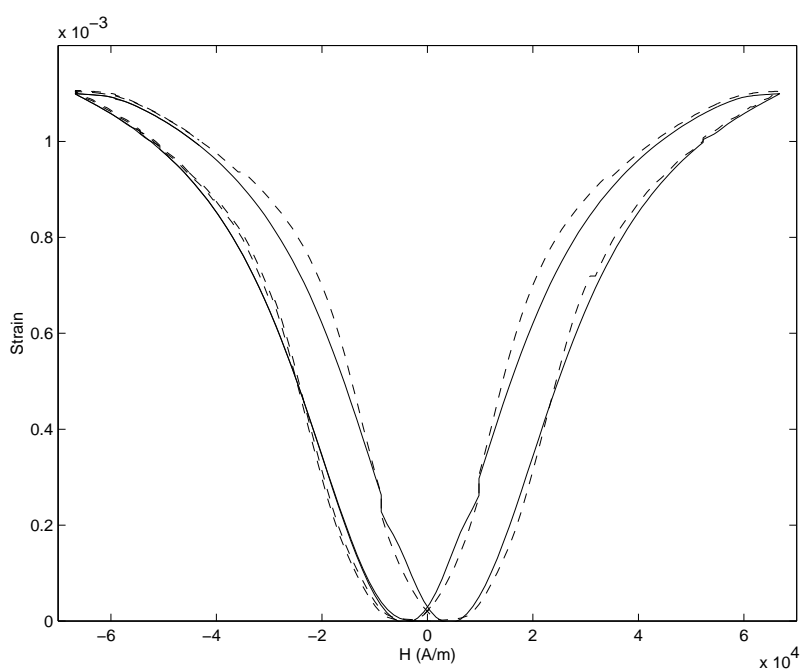

(b)

Figure 6. Experimental data $(---)$ and model dynamics (-) computed using filtered magnetic field data $H(t, x)=n I(t) \varphi(x) ;($ a) Magnetostriction $\lambda$, (b) Total strain $e$.

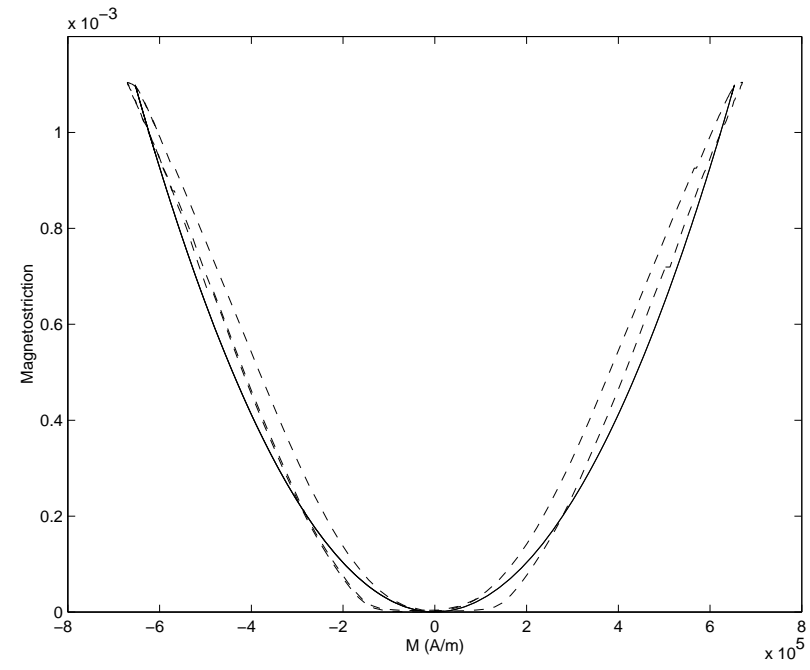

(a)

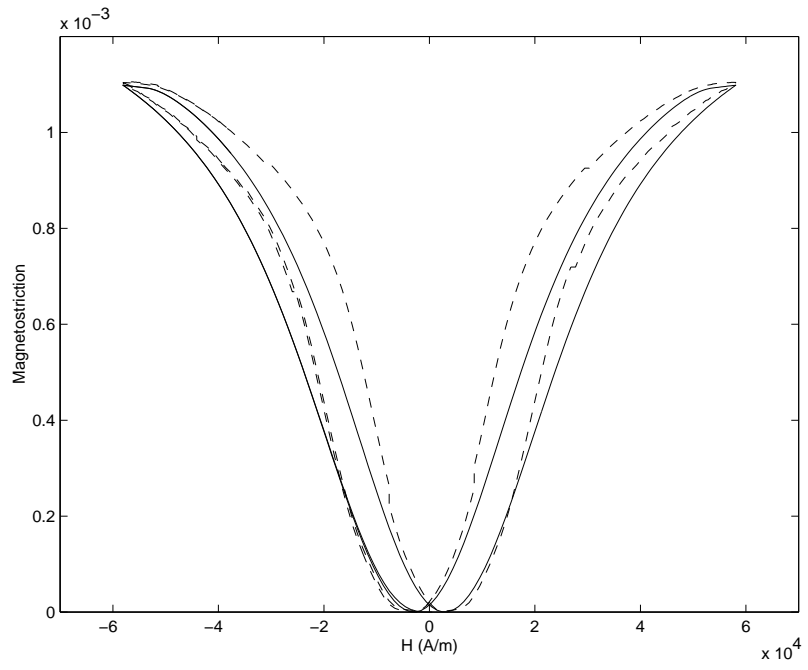

(b)

Figure 7. Experimental data (-- ) and model dynamics (-) computed using unfiltered magnetic field data $H(t)=n I(t)$ and scaled saturation magnetostriction $\lambda_{s}$; (a) $M-\lambda$ relation, (b) $H-\lambda$ relation.

\section{Concluding Remarks}

A dynamic model for strains generated by a magnetostrictive transducer in response to applied magnetic fields is presented in this paper. The external or applied strains are characterized through the ferromagnetic hysteresis theory of Jiles and Atherton combined with a quadratic rotation model. The internal or passive strains are quantified through a PDE model of the Terfenol rod dynamics. The combined model provided displacements and total strains which very accurately modeled experimental strain data measured under quasi-static conditions. We note that this approach for modeling the transducer output is similar in philosophy to that employed by Sablik and Jiles [6]. Our approach differs, however, in the manner through which physical parameters are specified and damping and spring 


\begin{tabular}{l|l|l|l}
\hline Magnetization Parameters & Structural Parameters & Spring and End Mass & Dimension \\
\hline$M_{s}=7.65 \times 10^{5} \mathrm{~A} / \mathrm{m}$ & $\rho=9250 \mathrm{~kg} / \mathrm{m}^{3}$ & $M_{L}=0.5 \mathrm{~kg}$ & $L=115 \mathrm{~mm}$ \\
$\lambda_{s}=1005 \times 10^{-6}$ & $E=3 \times 10^{10} \mathrm{~N} / \mathrm{m}^{2}$ & $k_{L}=2 \times 10^{6} \mathrm{~N} / \mathrm{m}$ & $d=12.7 \mathrm{~mm}$ \\
$\begin{array}{l}a=7012 \mathrm{~A} / \mathrm{m} \\
k=4000 \mathrm{~A} / \mathrm{m}\end{array}$ & $c_{D}=3 \times 10^{6} \mathrm{Ns} / \mathrm{m}^{2}$ & $c_{L}=1 \times 10^{3} \mathrm{Ns} / \mathrm{m}$ & \\
$\widetilde{\alpha}=-0.01$ & & & \\
$c=0.18$ & & & \\
\hline
\end{tabular}

Table 1. Physical parameters and dimensions employed in the magnetization and structural models.

characteristics are incorporated. It also differs through the use of a weak formulation of the model for subsequent approximation purposes.

The examples presented here demonstrate the accuracy of the model for characterizing major loop strain dynamics. Initial tests have demonstrated that the model also accurately characterizes harmonics due to the Terfenol rod. For example, the calculated model value of $1282 \mathrm{~Hz}$ for the rod primary frequency is within $5 \%$ of the measured value for the experimental system.

The current model is not fully coupled since it does not incorporate the effects of stress on the magnetization and magnetostriction in the rod. While the model incorporates certain dynamic effects, it must currently be restricted to low frequency regimes in which time-dependent $\mathrm{AC}$ losses are minimal. The extensions for including stress effects and eddy current losses are under investigation.

\section{Acknowledgements}

The authors express sincere appreciation to Tad Calkins and David Jiles for input regarding the modeling techniques employed here and to Brian Lund for his assistance in collecting the experimental data. The research of R.C.S. was supported in part by the Air Force Office of Scientific Research under the grant AFOSR F49620-95-10236. Financial support for M.J.D. and A.B.F. was provided by the NSF Young Investigator Award \#CMS9457288 of the Division of Civil and Mechanical Systems.

\section{References}

[1] D.L. Hall and A.B. Flatau, "Nonlinearities, harmonics and trends in dynamic applications of Terfenol-D," Proceedings of the SPIE Conference on Smart Structures and Intelligent Materials, Vol. 1917, Part 2, 1993, pp. 929939 .

[2] F.T. Calkins, R.C. Smith and A.B. Flatau, "An energy-based hysteresis model for magnetostrictive transducers," ICASE Report 97-60; IEEE Trans. Magn., submitted.

[3] D.C. Jiles, Introduction to Magnetism and Magnetic Materials, Chapman and Hall, New York, 1991.

[4] D.C. Jiles and D.L. Atherton, "Theory of ferromagnetic hysteresis," J. Magn. Magn. Mater., 61, pp. 48-60, 1986.

[5] D.C. Jiles, J.B. Thoelke and M.K. Devine, "Numerical determination of hysteresis parameters for the modeling of magnetic properties using the theory of ferromagnetic hysteresis," IEEE Trans. Magn., 28(1), pp. 27-35, 1992.

[6] M.J. Sablik and D.C. Jiles, "Coupled magnetoelastic theory of magnetic and magnetostrictive hysteresis," IEEE Trans. Magn., 29(3), pp. 2113-2123, 1993.

[7] B.D. Cullity, Introduction to Magnetic Materials, Addison-Wesley, Reading, MA, 1972. 
[8] F.T. Calkins, "Design, analysis, and modeling of giant magnetostrictive transducers," PhD Dissertation, Iowa State University, Ames, IA, 1997.

[9] R.C. Smith, "Modeling techniques for magnetostrictive actuators," Proceedings of the SPIE, Smart Structures and Materials 1997: Smart Structures and Integrated Systems, San Diego, CA, March 1997, Vol. 3041, pp. 243253.

[10] A.W. Leissa, Vibration of Plates, NASA SP-160, 1969, Reprinted by the Acoustical Society of America through the American Institute of Physics, 1993.

[11] C.L. Hom and N. Shankar, "Modeling the Dynamic Behavior of Electrostrictive Actuators," Proceedings of the SPIE, Smart Structures and Materials 1997: Smart Structures and Integrated Systems, San Diego, CA, March 1997, Vol. 3041, pp. 268-280.

[12] P.M. Prenter, Splines and Variational Methods, Wiley, New York, 1975. 